\title{
ÉTUDE OPTIQUE DE LA STRUCTURE JAHN TELLER DANS $\mathrm{BaTiO}_{3}$ DOPÉ
}

\author{
G. CHANUSSOT $(*)$, J. VOISIN, B. DEHAUT et G. GODEFROY (**)
}

Laboratoire de Diélectriques, Faculté des Sciences Mirande, 21000 Dijon, France

\begin{abstract}
Résumé. - Des expériences de thermophotocourants et d'absorption optique sur des cristaux de $\mathrm{BaTiO}_{3}$ montrent que les ions 3d coordinés octaédriquement jouent un rôle fondamental sur les propriétés diélectriques et structurales. Les expériences d'irradiation laser fournissent une alternative intéressante pour l'étude des systèmes vibroniques.
\end{abstract}

Abstract. - Thermophotocurrents experiments and optical absorption on $\mathrm{BaTiO}_{3}$ single crystals show that the octaedrically coordinated $3 \mathrm{~d}$ ions play a basic rôle in structural and dielectric properties. Laser irradiation provides an interesting way in studying vibronic systems.

1. Introduction. - Le but de ce travail est de montrer quelques résultats expérimentaux concernant les effets photoferroélectriques dans $\mathrm{BaTiO}_{3}$.

Par opposition aux transitions de phase photoinduite (irradiation continue au travers de la transition de phase) les effets photoferroélectriques (E. P. F.) correspondent à des irradiations limitées à une seule phase (paraélectrique ou ferroélectrique). Des résultats préliminaires ont déjà été publiés [1] ainsi que leur interprétation partielle [2] basée sur une théorie de couplage électron phonon (pseudo effet Jahn Teller - P. J. T.).

Notre plan d'études sera le suivant. Dans le paragraphe 2 nous décrivons une expérience typique d'E. P. F. ; nous montrerons que des cristaux dopés avec des ions $\mathrm{J}$. T. se comportent de façon très différente des cristaux dopés avec des ions $3 \mathrm{~d}$ habituellement non J. T. ; les effets d'un chauffage haute température sont clairement montrés (effet sur l'E. P. F. et sur la température de transition $T_{0}$ ). Dans 3 , nous établirons une connection entre $T_{0}$ et les gaps effectifs que l'on peut déterminer d'après les résultats d'absorption. Dans la conclusion 4, nous donnerons quelques principes généraux tirés de ces expériences qui ont permis (à Chanussot voir [3]) de déterminer avec précision le rôle de l'effet J. T. et de l'effet P. J. T. dans ce type d'expérience.

Les mesures utilisées sont des mesures pyroélectriques (réalisées par Dehaut) couplées à des mesures d'absorption (faite par Voisin). Les cristaux employés sont des cristaux Remeika (cristallisés par Godefroy) ; deux cristaux tirés (Sanders) ont servi de référence pour les études précédentes [4].

(*) Adresse à la date du Colloque : Bell Laboratories Murray Hill. New Jersey 07974 U. S. A.

(**) En l'absence de G. Chanussot communication présentée par G. Godefroy.
2. Effet photoferroélectrique (E. P. F.). - La figure 1 décrit un effet photoferroélectrique (E. P. F.) ; il correspond aux conditions d'irradiation :

$$
\begin{aligned}
& T_{\mathrm{i}}=T_{\mathrm{M}}-20\left({ }^{\circ} \mathrm{C}\right) \\
& t_{\mathrm{i}}=15 \mathrm{~min} \\
& P_{\mathrm{i}}=200 \mathrm{~mW} / \mathrm{cm}^{2} \text { (laser) } \\
& \lambda_{\mathrm{i}}=5145 \AA .
\end{aligned}
$$

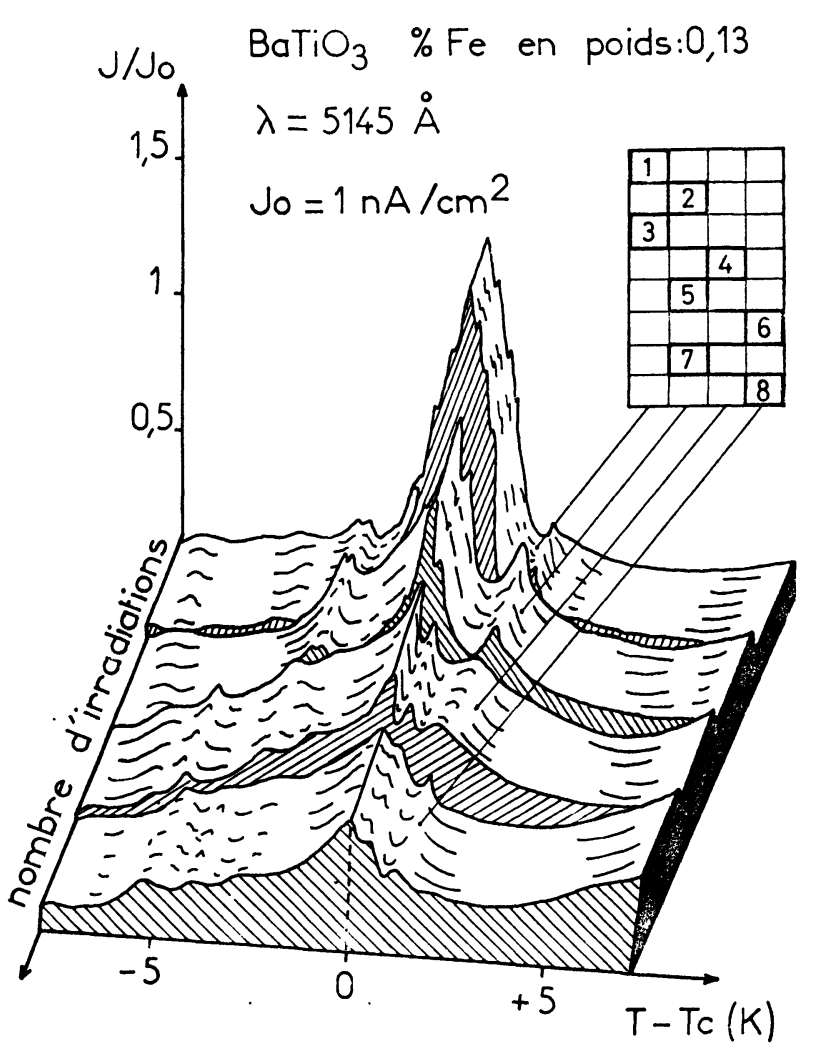

FIG. 1. - Etude des modifications du pyrocourant $J$ en fonction du nombre d'irradiation. 
Un champ $E_{\mathrm{p}}=0,5 \mathrm{kV} / \mathrm{cm}$ est appliqué dans la phase paraélectrique avant chaque mesure ; la température est alors descendue à $T_{0}-20\left({ }^{\circ} \mathrm{C}\right)$; à cette température la tension appliquée est ramenée à zéro. Après l'étape d'irradiation, le cristal est chauffé dans la phase paraélectrique $\left(T_{0}+10^{\circ} \mathrm{C}\right)$, alors un refroidissement est réalisé à nouveau avec un champ électrique appliqué le long de [100] $\left(0,5 \mathrm{kV} / \mathrm{cm}^{-1}\right)$. Les cristaux dopés au fer montrent une décroissance continue de polarisation quand les séquences précédentes sont répétées. En même temps la transition aiguë est changée en transition diffuse, comme pour une transition de phase photo-induite. La figure 2 représente la variation du maximum de $J$ présenté figure 1 en fonction du nombre d'irradiations. Le cas d'un cristal dopé au cuivre est représenté en même temps ( $f$ est le coefficient pyroélectrique calculé à partir de $J$ ).

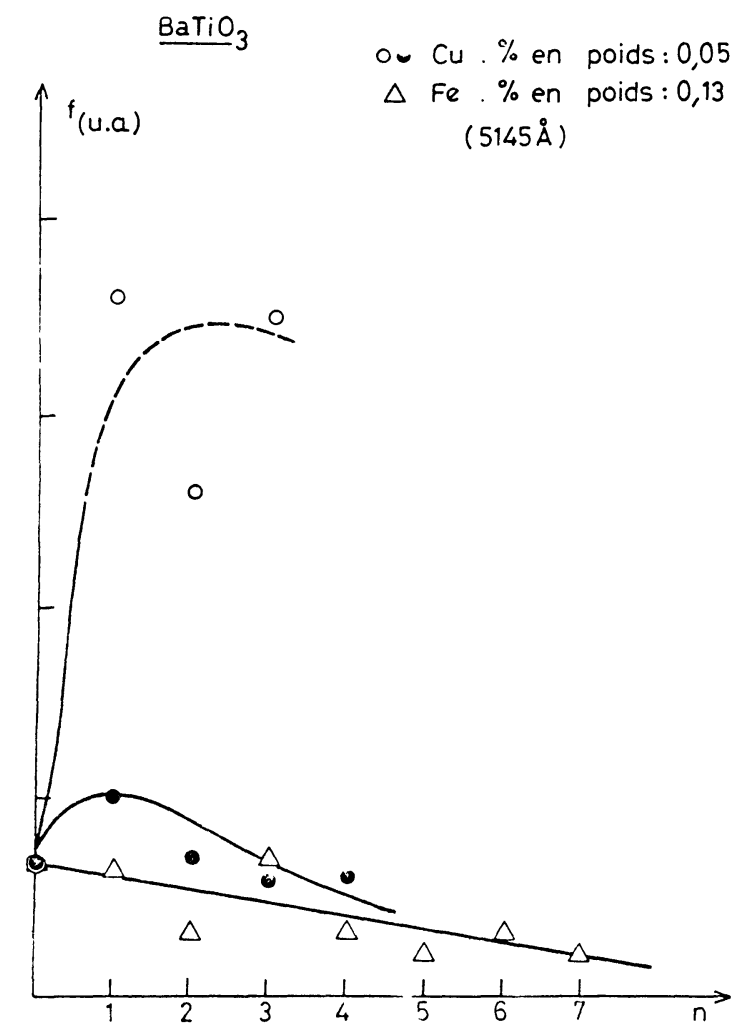

FIG. 2. - Variation du coefficient pyroélectrique maximum en fonction du nombre d'irradiation. Dans le cas du dopage au fer, les résultats sont déduits de la figure 1 .

Les variations de $f$ pour les cristaux dopés au $\mathrm{Fe}$ et au $\mathrm{Cu}$ montrent clairement un nouvel effet pour le cas du $\mathrm{Cu}$.

Le trappage dû au centre $\mathrm{V}$ peut être montré par un chauffage à haute température du cristal (Fig. 3). Ce rôle explique aussi pourquoi les cristaux dopés au Co ne montrent ni transition de phase photoinduite, ni effet photoferroélectrique aussi marqué que dans le cas du fer et du cuivre.

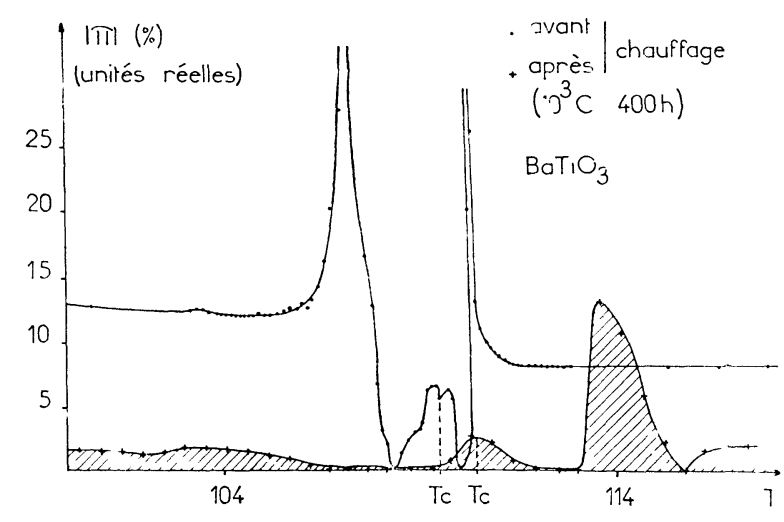

Fig. 3. - Effet du chauffage sur le pyrocourant $J$ :

$$
\pi=\frac{J \text { (après) }-J \text { (avant) }}{J \text { (après) }} \text {. }
$$

3. Le problème de la transition de phase. - Le chauffage modifie aussi la valeur de $T_{0}$ (Fig. 4): les contributions des effets isovalents et hétérovalents doivent donc être considérés en même temps.

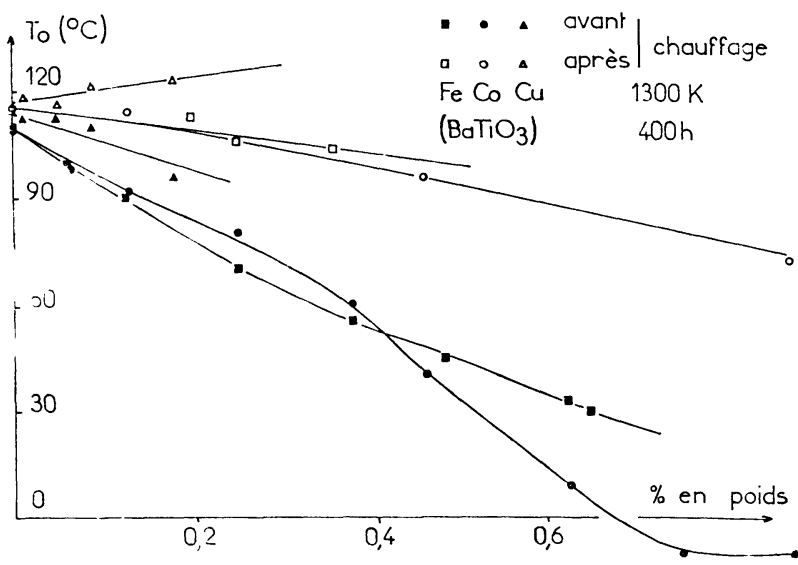

Fig. 4. - Variation de la température $T_{0}$ (maximum de $J$ et $f$ ) en fonction du dopage et du chauffage.

Afin de déterminer les rôles dus à l'effet P. J. T. et J. T. dans les cristaux dopés au fer, au cobalt et au cuivre (voir § 4), il a été intéressant de faire simultanément des mesures d'absorption :

Un gap unique ne peut pas être défini (Fig. 5). Les extrapolations des droites en pointillé donnent les gaps effectifs de 2,42 eV et 1,67 eV (approximativement les valeurs données dans la littérature, obtenues soit par mesures optiques, soit par mesures de conductivité).

4. Conclusion. - 4.1 Les cristaux dopés au $\mathrm{Fe}, \mathrm{Co}$, $\mathrm{Cu}$ sont considérés comme ayant les états de valence 3 , 2, 2. Les états de spin sont respectivement : haut, bas, bas. Ceci nous permet de déterminer dans quels cas un effet J. T. significatif $(E \otimes e)$ est susceptible d'apparaître ou de disparaître dans les expériences d'irradiation (voir [3]) (concept de centre J. T. optique). On a pu confirmer qu'il existe un spin haut $\mathrm{d}^{4}\left(\mathrm{Fe}^{3+}\right)$ et un ion $\mathrm{d}^{9}\left(\mathrm{Cu}^{2+}\right)$ avant irradiation. 


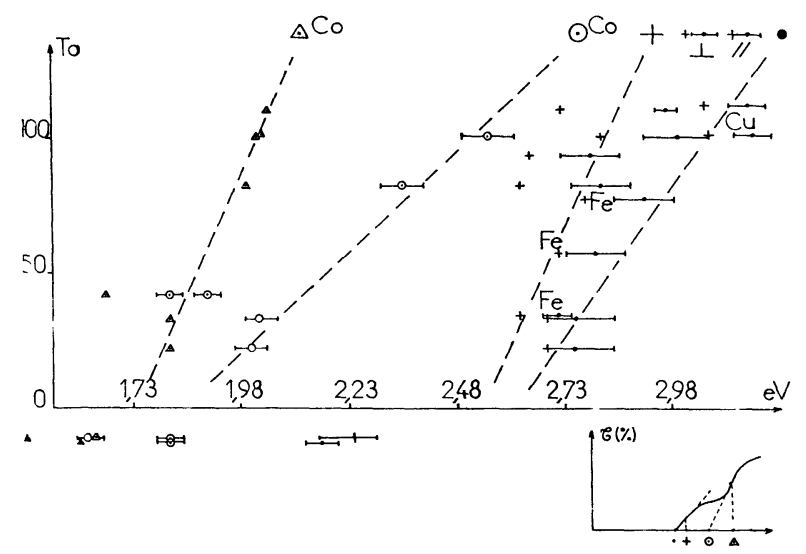

FIG. 5. - Représentation schématique des variations de $T_{0}$ et des gaps effectifs dans un cristal dopé : tous les points se réfèrent au Co, sauf indication contraire $\perp$ et $\|$ représentent les mesures sur le cristal tiré (polarisation de la lumière $\perp$ et $\|$ à l'axe $C$ ).

4.2 Pour les dopages les plus faibles $(\mathrm{Fe}, \mathrm{Cu})$, le mécanisme de compensation le plus probable est par centre $\mathrm{V}$; pour les dopages importants et pour les cristaux dopés au Co, la compensation se fait par ions $\mathrm{F}^{-}$. Il en résulte que l'effet le plus probable au niveau du défaut axial sera l'effet $P$. J. T. et non pas le J. T. Les effets J. T. et P. J. T. ont des effets dif- férents sur la structure en domaines parce que l'effet P. J. T. est orienté dans le réseau, alors que l'effet J. T. ne l'est pas. On observe toujours l'effet combiné de ces deux instabilités. Les résultats de la figure 1 et 2 s'interprètent respectivement avec les effets P. J.T. et J. T. [3], la dispersion par une intervention certaine de la structure en domaines.

4.3 Quand le gap décroît, les théories vibroniques donnent généralement un accroissement de $T_{0}$ : le contraire est précisément observé. Ceci implique que l'effet dominant (Fig. 4 et 5) soit dû aux changements de $W_{0}^{2}$. Les réactions chimiques à haute $T$ (évaporation de $\mathrm{TiF}^{4}$ et oxydation) montrent que $W_{0}^{2}$ est déterminé par les centres $\mathrm{V}$ et $\mathrm{F}^{-}$(voir aussi Fig. 3). ( $W_{0}$ caractérise les propriétés de vibration harmonique.)

4.4 Par suite de la contribution importante des effets thermiques, un troisième processus d'instabilité est susceptible de se produire conduisant à une répartition non uniforme des porteurs dans la bande polaronique de conduction, expliquant ainsi l'élargissement observé figure 1.

(Les effets des contraintes et d'une irradiation non uniforme semblent être exclus $a$ priori.)

\section{Bibliographie}

[1] Chanussot, G., Thiebaud, C., Ferroelectrics 8 (1974) 665.

[2] Chanussot, G., Ferroelectrics 8 (1974) 671.

[3] Chanussot, G., Symposium on Applications of Ferroelectrics Albuquerque, New Mexico (U. S. A.) 1975.
[4] Qu'il nous soit permis ici de remercier M. F. Micheron pour la fourniture des cristaux tirés (Thomson-CSF). 